\title{
Numerical Methods for Minimizers and Microstructures in Nonlinear Elasticity
}

\author{
Zhiping Li \\ Department of Mathematics, Peking University, \\ Beijing 100871, People's Republic of China
}

\begin{abstract}
A standard finite element method and a finite element truncation method are applied to solve the boundary value problems of nonlinear elasticity with certain nonconvex stored energy functions such as those of St. Venant-Kirchhoff materials. Finite element solutions are proved to exist and to be in the form of minimizers in appropriate sets of admissible finite element functions for both methods. Convergence of the finite element solutions to a solution in the form of a minimizer or microstructure for the boundary value problem is established. it is also shown that in the presence of Lavrentiev phenomenon in the problem the finite element truncation method can overcome the difficulty and converges to the absolute minimum while the standard finite element method converges to a poseudominimum which is a minimum in a slightly small set of admissible functions.
\end{abstract}

Subject classification : 65N12, 65N30, 35J60, 49J45, 73C50

Key words : numerical method, minimizer, microstructure, weak convergence, strong convergence, nonlinear elasticity

\section{Introduction}

In this paper, we consider numerical methods for the boundary value problems of nonlinear elasticity, or in mathematical terms the problem of minimizing the functional

$$
I(u)=\int_{\Omega} W(x, F) d x-\int_{\Omega} f \cdot u d x-\int_{\partial \Omega_{1}} g \cdot u d s
$$


in the set of admissible functions

$$
\begin{aligned}
A=\{u \in & W^{1, p}\left(\Omega ; R^{3}\right): \operatorname{adj} F \in L^{q}\left(\Omega ; M^{3}\right), \operatorname{det} F \in L^{r}(\Omega), \\
& \left.\operatorname{det} F>0, \text { a.e. in } \Omega, \text { and } u=u_{0} \text { on } \partial \Omega_{0}\right\},
\end{aligned}
$$

where $\Omega \subset R^{3}$ is a connected open set with Lipschitz continuous boundary $\partial \Omega, \partial \Omega_{0} \subset \partial \Omega, \partial \Omega_{1} \subset \partial \Omega$ with $\partial \Omega_{0} \cap \partial \Omega_{1}=\emptyset$ and area $\left(\partial \Omega_{0}\right) \neq 0$, $F=I+D u$ with $D u$ being the displacement gradient and $I$ being the $3 \times 3$ identity matrix, $a d j F$ is the transpose of the matrix of the cofactors of $F$ and $\operatorname{det} F$ is the determinant of $F, f$ is the body force and $g$ is the traction.

There is no general existence theorem for the problem. However, if the stored energy function $W(x, F)$ satisfies polyconvex conditions and certain growth and coerceiveness conditions, the minimum of $I(\cdot)$ in $A$ can be proved to be obtained (see [1] [2]) for any $f$ and $g$ satisfying the hypothesis

(c) $\Phi(u)=\int_{\Omega} f \cdot u d x+\int_{\partial \Omega_{1}} g \cdot u d s$ is continuous in $W^{1, p}\left(\Omega ; R^{3}\right)$.

The numerical methods for the corresponding problems have also been studied [3] [4] [5] [6].

In the case when $W(x, F)$ is not polyconvex, such as the case for St. Venant-Kirchhoff materials [7] where

$$
W(x, F) \equiv W^{*}(E)=\frac{\lambda}{2}(\operatorname{tr} E)^{2}+\mu \operatorname{tr} E^{2}
$$

with $E=\frac{1}{2}\left(F^{T} F-I\right)$ being the strain tensor and $\lambda>0, \mu>0$ being the Lamé parameters, the problem is more complicated. In the present paper, the following hypotheses recently introduced by Li [8] for stored energy functions $W(x, F)$ are considered.

(H1) There is a continuous function $G: \bar{\Omega} \times M^{3} \times M^{3} \times M^{3} \times R \rightarrow$ $R \cup\{+\infty\}$ such that $G(x, \cdot, \cdot, \cdot)$ is strictly convex and $W(x, F)=G(x, E, F, \operatorname{adj} F, \operatorname{det} F), \quad$ for all $x \in \bar{\Omega}$ and $F \in M_{+}^{3}$, where $M^{3}=\{$ all $3 \times 3$ matrices $\}, M_{+}^{3}=\left\{F \in M^{3}: \operatorname{det} F>0\right\}$, $E=\frac{1}{2}\left(F^{T} F-I\right)$.

(H2) There are constants $C_{0} \in R, C_{1}>0, s>1, p \geq 2 s, q \geq \frac{p}{p-1}$, $r>1$ such that

$$
G(x, E, F, H, \delta) \geq C_{0}+C_{1}\left(|E|^{s}+|F|^{p}+|H|^{q}+|\delta|^{r}\right),
$$

for all $(x, E, F, H, \delta) \in \bar{\Omega} \times M^{3} \times M^{3} \times M^{3} \times R_{+}$. 
(H3) $G(x, E, F, H, \delta)=+\infty$ if $\delta \leq 0$ and is finite elesewhere,

$$
\begin{array}{r}
G\left(x, E_{n}, F_{n}, H_{n}, \delta_{n}\right) \longrightarrow+\infty \\
\text { if }\left|E_{n}\right|+\left|F_{n}\right|+\left|H_{n}\right|+\left|\delta_{n}\right| \rightarrow+\infty \text { or } \delta_{n} \rightarrow 0^{+} .
\end{array}
$$

Li proved the following result.

Theorem 1.1 [8]. Let $W(x, F)$ satisfy the hypotheses (H1)-(H3). Let $f, g$ satisfy the hypothesis (c). Let $\left\{u_{j}\right\}$ be a minimizing sequence of $I(\cdot)$ in A. Suppose that $\inf _{v \in A} I(v)<+\infty$.

Then, (i): There exist a subsequence $\left\{u_{\nu}\right\}$ of $\left\{u_{j}\right\}$, function $u \in A$ and function $\hat{E} \in L^{s}\left(\Omega ; R^{3}\right)$ such that

$$
\begin{array}{rll}
u_{\nu} & \rightarrow u \quad \text { in } W^{1, p}\left(\Omega ; R^{3}\right), \\
\operatorname{adj} F_{\nu} & \rightarrow \operatorname{adj} F & \text { in } L^{q}\left(\Omega ; M^{3}\right), \\
\operatorname{det} F_{\nu} & \rightarrow \operatorname{det} F & \text { in } L^{r}(\Omega), \\
E_{\nu} & \rightarrow \hat{E} \quad \text { in } L^{s}\left(\Omega ; M^{3}\right),
\end{array}
$$

where $F_{\nu}=I+D u_{\nu}, E_{\nu}=\frac{1}{2}\left(F_{\nu} F_{\nu}-I\right)$, and

$$
I(\hat{E}, u) \leq \underline{\lim }_{\nu \rightarrow \infty} I\left(u_{\nu}\right)
$$

where

$$
I(\hat{E}, u)=\int_{\Omega} G(x, \hat{E}, F, a d j F, \operatorname{det} F) d x-\int_{\Omega} f \cdot u d x-\int_{\partial \Omega_{1}} g \cdot u d s .
$$

(ii): Either the weak convergence in (1.4) - (1.7) are in fact all strong convergence, in this case $u$ is a minimizer of $I(\cdot)$ in $A$ and $\hat{E}=\frac{1}{2}((I+$ $D u)^{T}(I+D u)-I$ ), or $\left\{u_{\nu}\right\}$ gives microtructure (see lemma 2.4 for the definition).

(iii): If the equality holds in (1.8) then

$$
\hat{E}=\frac{1}{2}\left((I+D u)^{T}(I+D u)-I\right)
$$

and $u$ is a minimizer of $I(\cdot)$ in $A$. Otherwise, $\left\{u_{\nu}\right\}$ gives microtructure.

Remark 1.1. Theorem 1.1 is an extension of Ball's result. In fact, if $G(x, \cdot, \cdot, \cdot, \cdot)$ does not explicitly depend on $E$, then the theorem simply reduces to the existence theorem for polyconvex materials [8] [9]. 
Remark 1.2. The result covers a large amount of nonlinear elastic materials including many homogeneous, isotropic materials whose stored energy functions are of the form

$$
W(x, F)=\frac{\lambda}{2}(\operatorname{tr} E)^{2}+\mu \operatorname{tr} E^{2}+o\left(|E|^{2}\right),
$$

where $o\left(|E|^{2}\right)$ is such that (H1)-(H3) are satisfied by $W(x, F)$ [8]. The theory can also be easily adjusted, roughly speaking by removing the restriction $\operatorname{det} F>0$ on the admissible functions and the hypothesis (H3) for $W(x, F)$, to cover St.Venant-Kirchhoff materials.

Our purpose is to show taht the minimizers and microstructures can be approximated by the minimizers of some finite problems obtained by applying certain finite element methods. First, a standard finite element method is applied to solve the problem. We will prove the existence of minimizers for the induced finite problem and discuss the convergence of the method in $\S 2$. Then, in $\S 3$, a finite element truncation method [11] is applied and the corresponding existence and convergence results are obtained. The results show that while the finite element truncation method can always produce a solution to the problem of minimizing $I(\cdot)$ in $A$, the standard finite element method may fail to do so, in fact it produces a solution to the problem of minimizing $I(\cdot)$ in $\hat{A}$, where $\hat{A}$ is in general a genuine subset of $A$ (see $\S 2$ ). As a consequence, the standard finite element method typically fail both to find the absolute minimum and to produce a solution to the original problem in which Lavrentiev phenonmenon [12] [13] occurs. To actually find numerically the minimizers of the corresponding finite problem is by no means trivial and is out of the scope of this paper.

\section{Existence and convergence of a standard finite element method}

As in a standard application of the finite element method, we introduce regular triangulations $T_{h}$ on $\Omega$ [10], where $h$ is the mesh size. For simplicity, we assume that $\bar{\Omega}=\cup_{K \in T_{h}} K, \partial \Omega_{0}$ consists of the faces of $T_{h}$ and $u(x)=0$ on $\partial \Omega_{0}$. Define

$$
\begin{aligned}
& \hat{A}_{h}=\left\{u \in C(\bar{\Omega}): u \text { is affine on each } K \in T_{h} ;\right. \\
& \left.u(x)=0, \forall x \in \partial \Omega_{0}, \operatorname{det}(I+D u)>0 \text {, a.e. in } \Omega\right\} .
\end{aligned}
$$

We consider the finite problems of minimizing the functional $I(\cdot)$ defined by (1.1) with $W(x, F)$ satisfying the hypotheses (H1) - (H3) and 
$f, g$ satisfying the hypothesis (c) (see $\S 1$ ) in the sets of admissible finite element functions $\hat{A}_{h}$.

Theorem 2.1 . For each fixed $h>0$, there exists a function $u_{h} \in \hat{A}_{h}$ such that

$$
I\left(u_{h}\right)=\inf _{v \in \hat{A}_{h}} I(v) .
$$

Proof. It is easily seen that $\hat{A}_{h} \neq \emptyset$ and $\inf _{v \in \hat{A}_{h}} I(v)<+\infty$. let $\left\{u_{j}\right\} \subset \hat{A}_{h}$ be a minimizing sequence of $I(\cdot)$ in $\hat{A}_{h}$. By (H2), (c) and the equivalence of the convergence in weak topology of $W^{1, p}\left(\Omega ; R^{3}\right)$ and that in strong topology of $W^{1, \infty}\left(\Omega ; R^{3}\right)$ in a finite dimensional function spaces [10], we conclude that there exist a subsequence of $\left\{u_{j}\right\}$, denoted again by $\left\{u_{j}\right\}$, and $u_{h} \in\left\{v \in C(\bar{\Omega}):\left.v\right|_{K}\right.$ is affine, $\left.\left.v\right|_{\partial \Omega_{0}}=0\right\}$ such that

$$
\begin{aligned}
& u_{j} \rightarrow u_{h} \quad \text { in } W^{1, \infty}\left(\Omega ; R^{3}\right), \\
& F_{j} \rightarrow F_{h} \quad \text { in } L^{\infty}\left(\Omega ; M^{3}\right), \\
& \operatorname{adj} F_{j} \rightarrow \operatorname{adj} F_{h} \text { in } L^{\infty}\left(\Omega ; M^{3}\right) \text {, } \\
& \operatorname{det} F_{j} \rightarrow \operatorname{det} F_{h} \text { in } L^{\infty}(\Omega) \text {, } \\
& E_{j} \rightarrow E_{h} \quad \text { in } L^{\infty}\left(\Omega ; M^{3}\right),
\end{aligned}
$$

where $F_{j}=I+D u_{j}, F_{h}=I+D u_{h}, E_{j}=\frac{1}{2}\left(F_{j}^{T} F_{j}-I\right)$ and $E_{h}=$ $\frac{1}{2}\left(F_{h}^{T} F_{h}-I\right)$. By $(\mathrm{H} 1)$ and $(\mathrm{c}), I(E, u)$ defined by $(1.9)$ is sequentially weakly lower semicontinuous [14]. Since $I\left(E_{j}, u_{j}\right)=I\left(u_{j}\right)$ for all $j$, we have

$$
I\left(E_{h}, u_{h}\right) \leq \underline{\lim }_{j \rightarrow \infty} I\left(u_{j}\right)=\inf _{v \in \hat{A}_{h}} I(v)<+\infty .
$$

This and (H3) imply that $\operatorname{det} F_{h}(x)>0$ for almost all $x \in \Omega$ and hence $u_{h} \in \hat{A}_{h} \subset A$. Thus $I\left(u_{h}\right)=I\left(E_{h}, u_{h}\right)$ and (2.2) follows.

To discuss the convergence of the finite element solutions, we introduce a different set of admissible functions $\hat{A}$. Let

$$
\begin{aligned}
A(j)=\{u \in & W^{1, \infty}\left(\Omega ; R^{3}\right) \cap A:|D u(x)| \leq j, \text { a.e. in } \Omega \\
& \text { and } \left.\operatorname{det}(I+D u(x)) \geq \frac{1}{j}, \text { a.e. in } \Omega\right\} .
\end{aligned}
$$

Define $d: A \times A \rightarrow R$ by

$$
d(u, v)=|u-v|_{1, p}+|I(u)-I(v)| .
$$

Define

$$
\hat{A}=\left\{u \in A: d\left(u, v_{j}\right) \rightarrow 0, \text { for a sequence } v_{j} \in A(j), j=1,2, \cdots\right\} .
$$

It is known that $\hat{A}$ is in general a genuine subset of $A$. 
Theorem 2.2. Let $h_{j}>0, j=1,2, \cdots$, and $\lim _{j \rightarrow \infty} h_{j}=0$. For each $j$, let $u_{j}$ be a minimizer of $I(\cdot)$ in $A_{h_{j}}$. Then, $\left\{u_{j}\right\}$ is a minimizing sequence of $I(\cdot)$ in $\hat{A}$.

Proof. By the definition of $\hat{A}$, it is sufficient to show that for any $M>1$ and $u \in A(M)$,

$$
\lim _{j \rightarrow \infty}\left|I(u)-I\left(u_{h_{j}}\right)\right|=0,
$$

where $u_{h_{j}}$ are the interpolations of $u$ in $A_{h_{j}}, j=1,2, \cdots$. But this follows directly from the fact [10] that

$$
\left\|u-u_{h_{j}}\right\|_{1, \infty} \rightarrow 0
$$

and hence also

$$
\left\|\operatorname{det}(I+D u)-\operatorname{det}\left(I+D u_{h_{j}}\right)\right\|_{\infty} \rightarrow 0,
$$

and the fact that the stored energy function $W(x, F)$ is continuous on $\bar{\Omega} \times M_{+}^{3}($ see $(\mathrm{H} 1))$.

Lemma 2.1 . Let $\left\{u_{j}\right\}$ be a minimizing sequence of $I(\cdot)$ in $\hat{A}$. Then, there exist a subsequence of $\left\{u_{j}\right\}$, denoted again by $\left\{u_{j}\right\}$, function $u \in A$ and function $\hat{E} \in L^{s}\left(\Omega ; M^{3}\right)$ such that

$$
\begin{aligned}
& u_{j} \rightarrow u \quad \text { in } W^{1, p}\left(\Omega ; R^{3}\right), \\
& F_{j} \rightarrow F \quad \text { in } L^{p}\left(\Omega ; M^{3}\right) \text {, } \\
& \operatorname{adj} F_{j} \rightarrow \operatorname{adj} F \text { in } L^{q}\left(\Omega ; M^{3}\right) \text {, } \\
& \operatorname{det} F_{j} \rightarrow \operatorname{det} F \text { in } L^{r}(\Omega) \text {, } \\
& E_{j} \rightarrow \hat{E} \quad \text { in } L^{s}\left(\Omega ; M^{3}\right),
\end{aligned}
$$

and

$$
I(\hat{E}, u) \leq \lim _{j \rightarrow \infty} I\left(u_{j}\right)=\inf _{v \in \hat{E}} I(v),
$$

where $I(\hat{E}, u)$ is defined by (1.9).

Proof. It follows from (H2), (c) and the sequentially weak continuity of Jacobians [1] [2] that (2.11) - (2.15) hold for a function $u \in$ $W^{1, p}\left(\Omega ; R^{3}\right)$ with $u=0$ on $\partial \Omega_{0}$ and function $\hat{E} \in L^{s}\left(\Omega ; M^{3}\right)$. (2.16) follows from the sequentially weakly lower semicontinuity of $I(\hat{E}, u)$ which is a consequnce of (H1) and (c) and a standard lower semicontinuity theorem [14]. Now, (H3) and (2.16) imply that $\operatorname{det} F>0$ for almost all $x \in \Omega$ and hence $u \in A$. 
Definition 2.1. A sequence of $\left\{u_{j}\right\} \subset \hat{A}$ is said to be a generalized solution to the problem of minimizing $I(\cdot)$ in $\hat{A}$, if it is a minimizing sequence of $I(\cdot)$ in $\hat{A}$, and (2.11) - (2.16) hold for some functions $u \in A$ and $\hat{E} \in L^{s}\left(\Omega ; R^{3}\right)$.

Thus, as a collorary of theorem 2.1, theorem 2.2 and lemma 2.1, we have

Theorem 2.3 . By solving the finite problem of minimizing $I(\cdot)$ in $\hat{A}_{h}, h>0$, we can obtain at least one generalized solution to the problem of minimizing $I(\cdot)$ in $\hat{A}$.

Next, we are to see what can be expected from a generalized solution.

Lemma 2.2 . Let $\left\{u_{j}\right\}$ be a generalized solution to the problem of minimizing $I(\cdot)$ in $\hat{A}$, and let $\left(u_{j}, E_{j}\right) \rightarrow(u, \hat{E})$ in $W^{1, p}\left(\Omega ; R^{3}\right) \times L^{s}\left(\Omega ; M^{3}\right)$. Suppose that

$$
I(u)=I(\hat{E}, u)=\lim _{j \rightarrow \infty} I\left(u_{j}\right)=\inf _{v \in \hat{A}} I(v) .
$$

Then

$$
\begin{aligned}
\hat{E} & =\frac{1}{2}\left((I+D u)^{T}(I+D u)-I\right), \\
u_{j} & \rightarrow u \quad \text { in } W^{1, p}\left(\Omega ; R^{3}\right), \\
E_{j} & \rightarrow \hat{E}=E \text { in } L^{s}\left(\Omega ; M^{3}\right),
\end{aligned}
$$

and $u$ is a minimizer of $I(\cdot)$ in $\hat{A}$.

Proof. The result follows from the same arguments as in [8] (see (b) and (c) in the proof of theorem 2.1 in [8] for the arguments).

Lemma 2.3 Let $\left\{u_{j}\right\}$ be a generalized solution to the problem of minimizing $I(\cdot)$ in $\hat{A}$, and let $\left(u_{j}, E_{j}\right) \rightarrow(u, \hat{E})$ in $W^{1, p}\left(\Omega ; R^{3}\right) \times L^{s}\left(\Omega ; M^{3}\right)$. Suppose that

$$
\hat{E}=\frac{1}{2}\left((I+D u)^{T}(I+D u)-I\right),
$$

and

$$
I(u)=I(\hat{E}, u)<\lim _{j \rightarrow \infty} I\left(u_{j}\right)=\inf _{v \in \hat{A}} I(v) .
$$

Then, Lavrentiev phenomenon [12] occurs in the problem, i.e.

$$
\inf _{v \in A} I(v)<\inf _{v \in \hat{A}} I(v) .
$$


Proof. The result is straight forward.

Lemma 2.4. Let $\left\{u_{j}\right\}$ be a generalized solution to the problem of minimizing $I(\cdot)$ in $\hat{A}$, and let $\left(u_{j}, E_{j}\right) \rightarrow(u, \hat{E})$ in $W^{1, p}\left(\Omega ; R^{3}\right) \times L^{s}\left(\Omega ; M^{3}\right)$. Suppose that

$$
\hat{E} \neq \frac{1}{2}\left((I+D u)^{T}(I+D u)-I\right),
$$

Then, $\left\{u_{j}\right\}$ gives microstructure, i.e. there exists a measurable subset $\hat{\Omega} \subset \Omega$ with meas $(\hat{\Omega})>0$ such that

$$
\begin{aligned}
\varliminf_{j \rightarrow \infty} \int_{\Omega^{\prime}}\left|D u_{j}-D u\right| d x & >0, \\
\lim _{j \rightarrow \infty} \int_{\Omega^{\prime}}\left(D u_{j}-D u\right) d x & =0,
\end{aligned}
$$

for any measurable subset $\Omega^{\prime} \subset \hat{\Omega}$ with meas $\left(\Omega^{\prime}\right)>0$.

Proof. Let

$$
\hat{\Omega}=\left\{x \in \Omega: \hat{E} \neq \frac{1}{2}\left((I+D u)^{T}(I+D u)-I\right)\right\} .
$$

By $(2.24)$, meas $(\hat{\Omega})>0$.

(2.26) is obvious, since $u_{j} \rightarrow u$, in $W^{1, p}\left(\Omega ; R^{3}\right)$.

Suppose that the lemma is not true. Then, there would exist a measurable subset $\Omega^{\prime} \subset \hat{\Omega}$ with meas $\left(\Omega^{\prime}\right)>0$, and a subsequence of $\left\{u_{j}\right\}$, denoted again by $\left\{u_{j}\right\}$, such that

$$
\lim _{j \rightarrow \infty} \int_{\Omega^{\prime}}\left|D u_{j}-D u\right| d x=0 .
$$

By extracting a further subsequence, we would then be able to find a subsequence $\left\{u_{\nu}\right\}$ of $\left\{u_{j}\right\}$ satisfying

$$
D u_{\nu} \rightarrow D u \quad \text { a.e. in } \Omega^{\prime},
$$

and thus

$$
E_{\nu} \rightarrow \frac{1}{2}\left((I+D u)^{T}(I+D u)-I\right) \text { a.e. in } \Omega^{\prime} .
$$

This would imply

$$
\hat{E}=\frac{1}{2}\left((I+D u)^{T}(I+D u)-I\right) \text { a.e. in } \Omega^{\prime},
$$

since $E_{\nu} \rightarrow \hat{E}$ in $L^{s}\left(\Omega ; M^{3}\right)$ and hence also in $L^{s}\left(\Omega^{\prime} ; M^{3}\right)$. But this contradicts the assumption that $\Omega^{\prime} \subset \hat{\Omega}$. 
Theorem 2.4 . Let $\left\{u_{j}\right\}$ be a generalized solution to the problem of minimizing $I(\cdot)$ in $\hat{A}$, and let $\left(u_{j}, E_{j}\right) \rightarrow(u, \hat{E})$ in $W^{1, p}\left(\Omega ; R^{3}\right) \times L^{s}\left(\Omega ; M^{3}\right)$.

Then, there are three possibilities

(i):

$$
I(\hat{E}, u)=\lim _{j \rightarrow \infty} I\left(u_{j}\right)=\inf _{v \in \hat{A}} I(v) .
$$

In this case,

$$
\begin{aligned}
\hat{E} & =\frac{1}{2}\left((I+D u)^{T}(I+D u)-I\right), \\
u_{j} & \rightarrow u \quad \text { in } W^{1, p}\left(\Omega ; R^{3}\right), \\
E_{j} & \rightarrow \hat{E}=E \text { in } L^{s}\left(\Omega ; M^{3}\right),
\end{aligned}
$$

and $u$ is a minimizer of $I(\cdot)$ in $\hat{A}$.

(ii):

$$
I(\hat{E}, u)<\lim _{j \rightarrow \infty} I\left(u_{j}\right)=\inf _{v \in \hat{A}} I(v)
$$

and

$$
\hat{E}=\frac{1}{2}\left((I+D u)^{T}(I+D u)-I\right) .
$$

In this case, Lavrentiev phenomenon occurs, i.e.

$$
\inf _{v \in A} I(v)<\inf _{v \in \hat{A}} I(v) .
$$

(iii):

$$
\hat{E} \neq \frac{1}{2}\left((I+D u)^{T}(I+D u)-I\right)
$$

In this case, $\left\{u_{j}\right\}$ gives microstructure.

Proof. It is easily seen that (i) - (iii) include all the possibilities. The conclusions in (i), (ii) and (iii) follows from directly from lemma 2.2 , lemma 2.3 and lemma 2.4 respectively.

The results in this section show that a standard application of finite element methods can be successful in producing regular solutions, but it may fail in general to produce a satisfactory result when a solution is singular.

\section{Existence and convergence of a finite el- ement truncation method}

In this section, we apply a finite element truncation method, which was designed to compute singular minimizers [11], to solve the problem of minimizing $I(\cdot)$ in $A$. 
Let $T_{h}$ be regular triangulations of $\Omega$ [10]. As in $\S 2$, for simplicity, assume that $\bar{\Omega}=\cup_{K \in T_{h}} K, \partial \Omega_{0}$ consists of faces of $T_{h}$ and $u(x)=0$ on $\partial \Omega_{0}$. Let

$$
\begin{aligned}
A_{h}=\{u \in C(\bar{\Omega}): & u \text { is affine on each } K \in T_{h}, \\
& \left.u(x)=0, \forall x \in \partial \Omega_{0}\right\} .
\end{aligned}
$$

Let $\left\{\alpha_{M}\right\}$ be an increasing sequence of positive numbers satisfying

$$
\lim _{M \rightarrow \infty} \alpha_{M}=+\infty .
$$

Define

$$
W_{M}(x, F)=\min \left\{W(x, F), \alpha_{M}\left(1+|F|^{p}\right)\right\}, \forall F \in M^{3} .
$$

We consider the finite problem of minimizing the functional

$$
I_{M}(u)=\int_{\Omega} W_{M}(x, F) d x-\int_{\Omega} f \cdot u d x-\int_{\partial \Omega_{1}} g \cdot u d s,
$$

with $W(x, F)$ satisfying hypotheses (H1) - (H3) and $f, g$ satisfying hypothesis (c) (see §1), in the set of admissible finite element functions $A_{h}$. We should notice that $A_{h}$ here is free from the restriction that $\operatorname{det}(I+D u(x))>0$ for almost all $x \in \Omega$. This makes the problem much easier to tackle.

Theorem 3.1. For each fixed $M \geq 1$ and $h>0$, there exists a $u_{h} \in A_{h}$ such that

$$
I_{M}\left(u_{h}\right)=\inf _{v \in A_{h}} I_{M}(v) .
$$

Proof. It is easily seen from (H1) and (3.3) that $W_{M}(x, F)$ is continuous on $A_{h}$ for each $M \geq 1$ and all $h>0$. Let $\left\{u_{j}\right\}$ be a minimizing sequence of $I_{M}(\cdot)$ in $A_{h}$. By the boundedness of $\left\|u_{j}\right\|_{1, p}$, which follows from (3.3) and (H2), the finite dimensionality of $A_{h}$ and continuity of $I_{M}(\cdot)$, which follows from the continuity of $W_{M}(x, F)$ and (c), we conclude that there exist a subsequence of $\left\{u_{j}\right\}$, denoted again by $\left\{u_{j}\right\}$, and function $u_{h} \in A_{h}$ such that

$$
\begin{gathered}
u_{j} \rightarrow u_{h}, \quad \text { in } W^{1, p}\left(\Omega ; R^{3}\right), \\
I_{M}\left(u_{h}\right)=\lim _{j \rightarrow \infty} I_{M}\left(u_{j}\right)=\inf _{v \in A_{h}} I_{M}(v) .
\end{gathered}
$$


Theorem 3.2. There exists a function $h(\cdot, \cdot): R_{+} \times R_{+} \rightarrow R_{+}$, which is nondecreasing with respect to the first variable and nonincreasing with respect to the second variable, such that for any $\epsilon>0$ and $M \geq 1$

$$
\inf _{v \in A_{h}} I_{M}(v)<\inf _{v \in A} I(v)+\epsilon,
$$

provided that $0<h<h(\epsilon, M)$.

Proof. Let $u \in A$ be such that

$$
I(u)<\inf _{v \in A} I(v)+\frac{\epsilon}{2} .
$$

Let $u_{h} \in A_{h}, h>0$ be such that

$$
\lim _{h \rightarrow 0}\left\|u_{h}-u\right\|_{1, p}=0 .
$$

Consider

$$
\begin{aligned}
& I_{M}\left(u_{h}\right)-I(u) \\
= & \int_{\Omega}\left(W_{M}\left(x, F_{h}\right)-W_{M}(x, F)\right) d x+\int_{\Omega}\left(W_{M}(x, F)-W(x, F)\right) d x \\
& +\int_{\Omega} f \cdot\left(u-u_{h}\right) d x+\int_{\partial \Omega_{1}} g \cdot\left(u-u_{h}\right) d s \\
= & I_{1}+I_{2}+I_{3}+I_{4} .
\end{aligned}
$$

By (c) and (3.9), there exists $h_{1}(\epsilon)>0$ such that

$$
\left|I_{3}+I_{4}\right|<\frac{\epsilon}{4}, \quad \forall h \in\left(0, h_{1}(\epsilon)\right) .
$$

It follows from (3.3) that

$$
I_{2}<0 .
$$

We claim that for any $\epsilon>0$ and $M \geq 1$ there exists $h_{2}(\epsilon, M)>0$ such that

$$
\left|I_{1}\right|<\frac{\epsilon}{4}, \quad \forall h \in\left(0, h_{2}(\epsilon, M)\right) .
$$

Suppose otherwise. Then, there would be $\epsilon_{0}>0, M_{0}>0$ and a decreasing sequence $\left\{h_{j}\right\}$ with $\lim _{j \rightarrow \infty} h_{j}=0$ such that

$$
\left|\int_{\Omega}\left(W_{M_{0}}\left(x, F_{j}\right)-W_{M_{0}}(x, F)\right) d x\right| \geq \frac{\epsilon_{0}}{4}, \forall j .
$$

By (3.9), we may assume without loss of generality that

$$
\begin{aligned}
& u_{j} \rightarrow u, \quad \text { a.e. in } \Omega \text {, } \\
& D u_{j} \rightarrow D u, \quad \text { a.e. in } \Omega \text {. }
\end{aligned}
$$


Thus, by the continuity of $W_{M_{0}}(\cdot, \cdot)$,

$$
W_{M_{0}}\left(x, F_{j}\right) \rightarrow W_{M_{0}}(x, F) \text {, a.e. in } \Omega .
$$

On the other hand, by (3.3),

$$
\left|W_{M_{0}}\left(x, F_{j}\right)-W_{M_{0}}(x, F)\right| \leq \alpha_{M_{0}}\left(2+\left|F_{j}\right|^{p}+|F|^{p}\right) .
$$

By (3.9), the right handside of (3.15) is uniformly integral continuous. Hence by (3.14) and (3.15)

$$
\lim _{j \rightarrow \infty} \int_{\Omega}\left|W_{M_{0}}\left(x, F_{j}\right)-W_{M_{0}}(x, F)\right| d x=0 .
$$

This is a contradiction.

Now, the theorem follows from (3.8) and (3.10) - (3.13) by taking $h(\epsilon, M)=\min \left\{h_{1}(\epsilon), h_{2}(\epsilon, M)\right\}$.

To obtain the convergence result, we first introduce the following lemmas.

Lemma 3.1 . Let $u_{M, h} \in A_{h}$ be such that

$$
I_{M}\left(u_{M, h}\right) \leq \inf _{v \in A_{h}} I_{M}(v)+1 .
$$

Let $\hat{\Omega}_{M, h}=\hat{\Omega}_{M}\left(u_{M, h}\right)$ where

$$
\hat{\Omega}_{M}(v)=\left\{x \in \Omega: W_{M}(x, F(x)) \neq W(x, F(x))\right\},
$$

where $F(x)=I+D v(x)$.

Then, there exist constants $M_{1} \geq 1$ and $C>0$ such that

$$
\left\|u_{M, h}\right\|_{1, p}^{p}+\left\|E_{M, h}\right\|_{s}^{s} \leq C, \quad \forall M \geq M_{1} \text { and } h>0,
$$

and

$$
\begin{aligned}
\left\|u_{M, h}\right\|_{1, p, \Omega_{M, h}}^{p}+\left\|\operatorname{adj} F_{M, h}\right\|_{q, \Omega_{M, h}}^{q} & +\left\|\operatorname{det} F_{M, h}\right\|_{r, \Omega_{M, h}}^{r}+\left\|E_{M, h}\right\|_{s, \Omega_{M, h}}^{s} \\
& \leq C, \quad \forall M \geq M_{1} \text { and } h>0,
\end{aligned}
$$

where $E_{M, h}=\frac{1}{2}\left(F_{M, h}^{T} F_{M, h}-I\right)$ and $\Omega_{M, h}=\Omega \backslash \hat{\Omega}_{M, h}$. Furthermore, let

$$
\hat{A}_{M, h}=\left\{u \in A_{h}: I_{M}(u) \leq \inf _{v \in A_{h}} I_{M}(v)+1\right\}
$$

and let

$$
\delta_{M, h}=\sup _{v \in \hat{A}_{M, h}} \operatorname{meas} \hat{\Omega}_{M}(v) .
$$

Then

$$
\delta_{M, h} \rightarrow 0, \quad \text { uniformly for } h>0 \text { as } M \rightarrow \infty .
$$


Proof. By (H1) and (3.2), there exists $M_{1} \geq 1$ such that

$$
\max _{x \in \bar{\Omega}} W(x, I) \leq \alpha_{M}, \quad \forall M \geq M_{1} .
$$

Thus, by (3.3)

$$
W_{M}(x, I)=W(x, I), \quad \forall x \in \bar{\Omega} \text { and } M \geq M_{1} .
$$

Since $u_{M, h}$ satisfies (3.16), (3.21) implies that

$$
I_{M}\left(u_{M, h}\right) \leq I_{M}(0)+1=I(0)+1, \quad \forall M \geq M_{1} \text { and } h>0 .
$$

By (H2) and (3.3), we have

$$
W_{M}(x, F) \geq \hat{C}_{0}+\hat{C}_{1}|F|^{p}, \quad \forall x \in \bar{\Omega} \text { and } F \in M^{3},
$$

for some constants $\hat{C}_{0} \in R$ and $\hat{C}_{1}>0$.

(3.22), (3.23) together with (c) and Poincaré inequality [15] give

$$
\left\|u_{M, h}\right\|_{1, p}^{p} \leq \hat{C}, \quad \forall M \geq M_{1} \text { and } h>0,
$$

where $\hat{C}$ is a constant. Since $2 s \leq p,(3.24)$ implies (3.18).

It follows from (H2), (3.3), (3.22) and the positiveness of $W_{M}\left(x, F_{M, h}\right)$ on $\hat{\Omega}_{M, h}$ that

$$
\begin{aligned}
& \int_{\Omega_{M, h}}\left[C_{0}+C_{1}\left(\left|F_{M, h}\right|^{p}+\left|\operatorname{adj} F_{M, h}\right|^{q}+\left|\operatorname{det} F_{M, h}\right|^{r}+\left|E_{M, h}\right|^{s}\right)\right] d x \\
& \leq \int_{\Omega_{M, h}} W\left(x, F_{M, h}\right) d x=\int_{\Omega_{M, h}} W_{M}\left(x, F_{M, h}\right) d x \\
& \leq I_{M}\left(u_{M, h}\right)+\int_{\Omega} f \cdot u_{M, h} d x+\int_{\partial \Omega_{1}} g \cdot u_{M, h} d s \\
& \leq I(0)+1+\int_{\Omega} f \cdot u_{M, h} d x+\int_{\partial \Omega_{1}} g \cdot u_{M, h} d s, \\
& \quad \forall M \geq M_{1} \text { and } h>0 .
\end{aligned}
$$

Thus, by (c) and (3.24), we have (3.19).

For any $u_{M, h} \in A_{h}$ satisfying (3.16), it follows from (3.3), (3.17) and (3.22) that

$$
\begin{aligned}
& \alpha_{M} \text { meas } \hat{\Omega}_{M, h} \leq \int_{\hat{\Omega}_{M, h}} W_{M}\left(x, F_{M, h}\right) d x \\
= & I_{M}\left(u_{M, h}\right)-\int_{\Omega_{M, h}} W_{M}\left(x, F_{M, h}\right) d x+ \\
& +\int_{\Omega} f \cdot u_{M, h} d x+\int_{\partial \Omega_{1}} g \cdot u_{M, h} d s
\end{aligned}
$$




$$
\begin{gathered}
\leq I(0)+1-\int_{\Omega_{M, h}} W\left(x, F_{M, h}\right) d x+ \\
+\int_{\Omega} f \cdot u_{M, h} d x+\int_{\partial \Omega_{1}} g \cdot u_{M, h} d s, \\
\forall M \geq M_{1} \text { and } h>0 .
\end{gathered}
$$

Thus, (3.20) follows from (H2), (c), (3.18), (3.19) and (3.2).

Lemma 3.2 . Let $\left\{M_{j}\right\}$ be such that

$$
\begin{aligned}
& \lim _{j \rightarrow \infty} M_{j}=+\infty \\
& \sum_{j=1}^{\infty} \delta_{M_{j}}<+\infty
\end{aligned}
$$

where $\delta_{M}=\sup _{h>0} \sup _{v \in \hat{A}_{M, h}}$ meas $\hat{\Omega}_{M}(v)$ with

$$
\hat{A}_{M, h}=\left\{u \in A_{h}: I_{M}(u) \leq \inf _{v \in A_{h}} I_{M}(v)+1\right\}
$$

and $\hat{\Omega}_{M}(v)$ being defined by (3.17). Let $h_{j} \in R_{+}$and $u_{j} \in A_{h_{j}}$ be such that

$$
I_{M_{j}}\left(u_{j}\right) \leq \inf _{v \in A_{h_{j}}} I_{M_{j}}(v)+1
$$

Then, there exist a subsequence $\left\{u_{\nu}\right\}$ of $\left\{u_{j}\right\}$, decreasing measurable subsets $\Omega_{l} \subset \Omega$, functions $u \in W^{1, p}\left(\Omega ; R^{3}\right)$ with $u=0$ on $\partial \Omega_{0}$ and $\hat{E} \in L^{s}\left(\Omega ; M^{3}\right)$ such that

$$
\begin{aligned}
& \lim _{l \rightarrow \infty} \text { meas } \Omega_{l}=0, \\
u_{\nu} & \rightarrow u \quad \text { in } W^{1, p}\left(\Omega ; R^{3}\right), \\
\operatorname{adj} F_{\nu} & \rightarrow \operatorname{adj} F \text { in } L^{q}\left(\Omega \backslash \Omega_{l} ; M^{3}\right), \text { for each fixed } l, \\
\operatorname{det} F_{\nu} & \rightarrow \operatorname{det} F \text { in } L^{r}\left(\Omega \backslash \Omega_{l}\right), \text { for each fixed } l, \\
E_{\nu} & \rightarrow \hat{E} \quad \text { in } L^{s}\left(\Omega ; M^{3}\right),
\end{aligned}
$$

Proof. Let

$$
\Omega_{l}=\cup_{j=l}^{\infty} \hat{\Omega}_{j},
$$

where $\hat{\Omega}_{j}=\hat{\Omega}_{M_{j}}\left(u_{j}\right)$ is defined by (3.17). It is obvious by the definition and (3.26) that $\Omega_{l}$ are decreasing and satisfy (3.28).

By lemma 3.1, $\left(F_{j}\right.$, adj $F_{j}$, det $\left.F_{j}, E_{j}\right)$ are bounded in $L^{p}\left(\Omega ; M^{3}\right) \times$ $L^{q}\left(\Omega \backslash \hat{\Omega}_{j} ; M^{3}\right) \times L^{r}\left(\Omega \backslash \hat{\Omega}_{j}\right) \times L^{s}\left(\Omega ; M^{3}\right)$ for each $j$ and the bounds can 
be chosen to be independent of $j$, and hence they are uniformly bounded in $L^{p}\left(\Omega ; M^{3}\right) \times L^{q}\left(\Omega \backslash \hat{\Omega}_{l} ; M^{3}\right) \times L^{r}\left(\Omega \backslash \hat{\Omega}_{l}\right) \times L^{s}\left(\Omega ; M^{3}\right)$ for all $j \geq l$.

Extracting a subsequence $\left\{u_{\nu}\right\}$ form $\left\{u_{j}\right\}$ by applying the diagonal process and making use of the weak continuity of Jacobians [1] [2], we obtain $(3.29)-(3.32)$.

Lemma 3.3 . Let $\Psi: \Omega \times R^{m} \times R^{n} \rightarrow R \cup\{+\infty\}$ satisfy

(i) $\Psi$ is an extended continuous function,

(ii) $\Psi(x, u, \cdot)$ is convex,

(iii) $\Psi(x, u, P) \geq C$, for some constant $C \in R$.

Let $\Psi_{M}: \Omega \times R^{m} \times R^{n} \rightarrow R \cup\{+\infty\}$ satisfy

(1) $\Psi_{M}$ is an extended continuous function,

(2) $\Psi_{M} \rightarrow \Psi$ locally uniformly in $\Omega \times R^{m} \times R^{n}$, i.e. there exists a sequence of measurable subsets $\Omega_{i} \subset \Omega$ with $\lim _{i \rightarrow \infty}$ meas $\left(\Omega \backslash \Omega_{i}\right)=$ 0 such that for each fixed $i$ and any compact subset $G \subset R^{m} \times R^{n}$$$
\Psi_{M}(x, u, P) \rightarrow \Psi(x, u, P), \quad \text { uniformly on } \Omega_{i} \times G \text { as } M \rightarrow+\infty \text {, }
$$

(3) $\Psi_{M}(x, u, P) \geq C$, for some constant $C \in R$.

Let $1 \leq p_{1} \leq+\infty, 1 \leq p_{2} \leq+\infty$, and let $\left\{u_{M}\right\}, u \in L^{p_{1}}\left(\Omega ; R^{m}\right)$ and $\left\{P_{M}\right\}, P \in L^{p_{2}}\left(\Omega ; R^{n}\right)$ be such that

$$
\begin{aligned}
& u_{M} \rightarrow u \quad \text { in } L^{p_{1}}\left(\Omega ; R^{m}\right), \\
& P_{M} \rightarrow P \quad \text { in } L^{p_{2}}\left(\Omega ; R^{n}\right) .
\end{aligned}
$$

Then

$$
\int_{\Omega} \Psi(x, u, P) d x \leq \underline{\lim }_{M \rightarrow \infty} \int_{\Omega} \Psi_{M}\left(x, u_{M}, P_{M}\right) d x .
$$

Lemma 3.3 is a special case of a general lower semicontinuity theorem given by $\mathrm{Li}[16]$, where the theorem is proved under weaker hypotheses for $\Psi$ and $\Psi_{M}$.

Theorem 3.3 . Let $\epsilon_{j}>0$ satisfy $\lim _{j \rightarrow \infty} \epsilon_{j}=0$. Let $M_{j}$ satisfy (3.25) and (3.26). Let $0<h_{j}<h\left(\epsilon_{j}, M_{j}\right)$, where $h(\cdot, \cdot)$ is determined by theorem 3.2. Let $u_{j} \in A_{h_{j}}$ be such that

$$
I_{M_{j}}\left(u_{j}\right) \leq \inf _{v \in A_{h_{j}}} I_{M_{j}}(v)+\epsilon_{j} .
$$


Then, there exist a subsequence $\left\{u_{\nu}\right\}$ of $\left\{u_{j}\right\}$, decreasing measurable subsets $\Omega_{l} \subset \Omega$, functions $u \in A$ and $\hat{E} \in L^{s}\left(\Omega ; M^{3}\right)$ such that (3.28) (3.32) hold and

$$
I(\hat{E}, u) \leq \lim _{j \rightarrow \infty} I_{M_{j}}\left(u_{j}\right)=\inf _{v \in A} I(v)
$$

where $I(\hat{E}, u)$ is defined by (1.9).

Proof. It follows from lemma 3.2 that (3.28) - (3.32) hold for some decreasing measurable subsets $\Omega_{l} \subset \Omega$, functions $u \in W^{1, p}\left(\Omega ; R^{3}\right)$ with $u=0$ on $\partial \Omega_{0}$ and $\hat{E} \in L^{s}\left(\Omega ; M^{3}\right)$.

It is easily seen that $W(x, P)$ and $W_{M}(x, P)$ satisfy (i) - (iii) and (1) - (3) in lemma 3.3 respectively. Thus by lemma 3.3, hypothesis (c) (see $\S 1$ ), we have

$$
\begin{aligned}
& \int_{\Omega \backslash \Omega_{l}} W(x, \hat{E}, F) d x-\int_{\Omega} f \cdot u d x-\int_{\partial \Omega_{1}} g \cdot u d s \\
\leq & \underline{\lim }_{\nu \rightarrow \infty}\left(\int_{\Omega \backslash \Omega_{l}} W_{M_{\nu}}\left(x, F_{M_{\nu}}\right) d x-\int_{\Omega} f \cdot u_{M_{\nu}} d x-\int_{\partial \Omega_{1}} g \cdot u_{M_{\nu}} d s\right) \\
= & \underline{\lim }_{\nu \rightarrow \infty}\left(I_{M_{\nu}}\left(u_{\nu}\right)-\int_{\Omega_{l}} W_{M_{\nu}}\left(x, F_{M_{\nu}}\right) d x\right),
\end{aligned}
$$

where $W(x, \hat{E}, F)=G(x, \hat{E}, F, \operatorname{adj} F$, $\operatorname{det} F)($ see $(\mathrm{H} 1))$.

By (H2) and (3.3), there is a constant $\hat{C}_{0} \geq 0$ such that

$$
-W_{M_{\nu}}\left(x, F_{\nu}\right) \leq \hat{C}_{0}
$$

Thus, by theorem 3.2, (3.33) and (3.35), we have

$$
\begin{aligned}
& \int_{\Omega \backslash \Omega_{l}} W(x, \hat{E}, F) d x-\int_{\Omega} f \cdot u d x-\int_{\partial \Omega_{1}} g \cdot u d s \\
\leq & \inf _{v \in A} I(v)+\hat{C}_{0} \text { meas } \Omega_{l}, \quad \forall l .
\end{aligned}
$$

This and (H3) imply that

$$
\operatorname{det} F>0 \text { a.e. in } \Omega \backslash \Omega_{l}, \quad \forall l,
$$

and thus, by (3.28)

$$
\operatorname{det} F>0 \text { a.e. in } \Omega \text {. }
$$

Hence we have $u \in A$.

Let $l \rightarrow+\infty$ in (3.36), noticing that $W(x, \hat{E}, F)$ is bounded from below by a constant and by passing to limit, we obtain (3.34). 
Lemma 3.4 (see [9]). Let $\Psi_{j}, \Psi, \xi_{j}, \xi, \eta_{j}, \eta \in L^{1}(\Omega)$ with $\xi_{j} \geq \Psi_{j} \geq \eta_{j}$ for all $j$. Suppose that $\Psi_{j} \rightarrow \Psi, \xi_{j} \rightarrow \xi, \eta_{j} \rightarrow \eta$, a.e. in $\Omega$, and $\int_{\Omega} \xi_{j} d x \rightarrow \int_{\Omega} \xi d x, \int_{\Omega} \eta_{j} d x \rightarrow \int_{\Omega} \eta d x$, as $j \rightarrow \infty$.

Then

$$
\int_{\Omega} \Psi_{j} d x \rightarrow \int_{\Omega} \Psi d x, \quad \text { as } j \rightarrow \infty .
$$

Proof. The result follows by applying Fatou's lemma to the sequences $\xi_{j}-\Psi_{j}$ and $\Psi_{j}-\eta_{j}$.

Lemma 3.5 Let $\Omega^{\prime} \subset \Omega$. Let $\left\{u_{\nu}\right\}$, u be such that

$$
\begin{aligned}
u_{\nu} & \rightarrow u \quad \text { in } W^{1, p}\left(\Omega^{\prime} ; R^{3}\right), \\
\operatorname{adj} F_{\nu} & \rightarrow \text { adj } F \text { in } L^{q}\left(\Omega^{\prime} ; M^{3}\right) \\
\operatorname{det} F_{\nu} & \rightarrow \operatorname{det} F \text { in } L^{r}\left(\Omega^{\prime}\right), \\
E_{\nu} & \rightarrow E \quad \text { in } L^{s}\left(\Omega^{\prime} ; M^{3}\right),
\end{aligned}
$$

and

$$
\int_{\Omega^{\prime}} W(x, F) d x=\lim _{\nu \rightarrow+\infty} \int_{\Omega^{\prime}} W\left(x, F_{\nu}\right) d x<+\infty .
$$

Then

$$
\begin{aligned}
u_{\nu} & \rightarrow u \quad \text { in } W^{1, p}\left(\Omega^{\prime} ; R^{3}\right), \\
\operatorname{adj} F_{\nu} & \rightarrow \operatorname{adj} F \text { in } L^{q}\left(\Omega^{\prime} ; M^{3}\right) \\
\operatorname{det} F_{\nu} & \rightarrow \operatorname{det} F \text { in } L^{r}\left(\Omega^{\prime}\right), \\
E_{\nu} & \rightarrow E \quad \text { in } L^{s}\left(\Omega^{\prime} ; M^{3}\right) .
\end{aligned}
$$

The result follows from a standard argument (see [9]) that, roughly speaking, for a strictly convex integral functional $J(v)$ satisfying certain coerceivness hypothesis, such as (H2) in this case, $v_{j} \rightarrow v$ and $J\left(v_{j}\right) \rightarrow$ $J(v)$ imply $v_{j} \rightarrow v$. For details of the argument see [9] or (b) in the proof of theorem 2.1 in [8].

Theorem 3.4. Let $\epsilon_{j}, M_{j}, h_{j}$ and $u_{j} \in A_{h_{j}}$ satisfy the same conditions as in theorem 3.3. Let $\left\{u_{\nu}\right\} \subset\left\{u_{j}\right\}, \Omega_{l}=\cup_{j=l}^{\infty} \hat{\Omega}_{M_{j}}\left(u_{j}\right)$ with $\hat{\Omega}_{M_{j}}\left(u_{j}\right)$ being defined by (3.17), $u \in A$ and $\hat{E} \in L^{s}\left(\Omega ; M^{3}\right)$ be such that (3.28) (3.32) and (3.34) hold.

Then, either

(a):

$$
\hat{E}=\frac{1}{2}\left((I+D u)^{T}(I+D u)-I\right) .
$$


In this case, $u \in A$ is a minimizer of $I(\cdot)$ in $A$, and we have

$$
\begin{aligned}
& u_{\nu} \rightarrow u \quad \text { in } W^{1, p}\left(\Omega ; R^{3}\right), \\
& \operatorname{adj} F_{\nu} \rightarrow \operatorname{adj} F \quad \text { in } L^{q}\left(\Omega \backslash \Omega_{l} ; M^{3}\right) \text {, for each } l \text {, } \\
& \operatorname{det} F_{\nu} \rightarrow \operatorname{det} F \quad \text { in } L^{r}\left(\Omega \backslash \Omega_{l}\right) \text {, for each } l \text {, } \\
& E_{\nu} \rightarrow E=\hat{E} \quad \text { in } L^{s}\left(\Omega ; M^{3}\right) \text {. }
\end{aligned}
$$

or

(b):

$$
\hat{E} \neq \frac{1}{2}\left((I+D u)^{T}(I+D u)-I\right) .
$$

In this case, $\left\{u_{\nu}\right\}$ gives microstructure.

Proof. In case (a), it follows from (3.34) that

$$
I(u)=\inf _{v \in A} I(v)
$$

a.e. $u \in A$ is a minimizer of $I(\cdot)$ in $A$.

Further, let $\Omega^{\prime} \subset \Omega \backslash \Omega_{l}$ for some $l$ be a measurable subset of $\Omega$. Then, it follows from lemma 3.3 and (3.38) that

$$
\int_{\Omega^{\prime}} W(x, F) d x \leq \underline{\lim }_{\nu \rightarrow \infty} \int_{\Omega^{\prime}} W_{M_{\nu}}\left(x, F_{\nu}\right) d x .
$$

This implies

$$
\int_{\Omega \backslash \Omega_{l}} W(x, F) d x=\underline{\lim }_{\nu \rightarrow \infty} \int_{\Omega \backslash \Omega_{l}} W_{M_{\nu}}\left(x, F_{\nu}\right) d x, \text { for each } l .
$$

Since otherwise, there would be some $l_{0} \geq 1$ such that

$$
\int_{\Omega \backslash \Omega_{l_{0}}} W(x, F) d x-\underline{\lim }_{\nu \rightarrow \infty} \int_{\Omega \backslash \Omega_{l_{0}}} W_{M_{\nu}}\left(x, F_{\nu}\right) d x=\delta<0,
$$

and thus we would have

$$
\begin{aligned}
\int_{\Omega \backslash \Omega_{l}} W(x, F) d x & =\int_{\Omega_{l_{0}} \backslash \Omega_{l}} W(x, F) d x+\int_{\Omega \backslash \Omega_{l_{0}}} W(x, F) d x \\
& \leq \underline{\lim }_{\nu \rightarrow \infty} \int_{\Omega \backslash \Omega_{l}} W_{M_{\nu}}\left(x, F_{\nu}\right) d x+\delta .
\end{aligned}
$$

Hence, by passing to limit, we would have

$$
I(u) \leq \inf _{v \in A} I(v)+\delta<\inf _{v \in A} I(v) .
$$

This contradicts (3.44). 
By the definition of $\Omega_{l}$ and (3.46), we have

$$
\int_{\Omega \backslash \Omega_{l}} W(x, F) d x=\underline{\lim }_{\nu \rightarrow \infty} \int_{\Omega \backslash \Omega_{l}} W\left(x, F_{\nu}\right) d x \text {. for each } l
$$

Thus, (3.29) - (3.42), (3.38) and lemma3.5 imply (3.40), (3.41) and

$$
\begin{array}{ll}
u_{\nu} \rightarrow u, \quad \text { in } W^{1, p}\left(\Omega \backslash \Omega_{l} ; R^{3}\right), & \text { for each } l, \\
E_{\nu} \rightarrow E=\hat{E}, \quad \text { in } L^{s}\left(\Omega \backslash \Omega_{l} ; M^{3}\right), & \text { for each } l .
\end{array}
$$

By (3.40), (3.41), (3.48) and (3.49), we can extract a further subsequence of $\left\{u_{\mu}\right\}$, again denoted by $\left\{u_{\mu}\right\}$, such that

$$
\left(u_{\mu}, \operatorname{adj} F_{\mu}, \operatorname{det} F_{\mu}, E_{\mu}\right) \rightarrow(u, \operatorname{adj} F, \operatorname{det} F, E), \quad \text { a.e. in } \Omega .
$$

Thus, by (3.3), we also have

$$
W_{M_{\mu}}\left(x, F_{\mu}(x)\right) \rightarrow W(x, F(x)) \text {, a.e. in } \Omega .
$$

On the other hand, it follows from (H2) and (3.3) that there is a constant $C>0$ such that

$$
\begin{aligned}
0 & \leq\left|F_{\mu}(x)-F(x)\right|^{p}+\left|E_{\mu}(x)-E(x)\right|^{s} \\
& \leq C\left(1+W_{M_{\mu}}\left(x, F_{\mu}(x)\right)+W(x, F(x))\right), \forall \mu .
\end{aligned}
$$

Now, it follows from (3.34), (3.44), (3.50) - (3.52) and lemma 3.4 that

$$
\left\|F_{\mu}-F\right\|_{p}^{p}-\left\|E_{\mu}-E\right\|_{s}^{s} \rightarrow 0, \quad \text { as } \mu \rightarrow \infty .
$$

This gives (3.39) and (3.42).

In case (b), $\left\{u_{\nu}\right\}$ gives microstructure on

$$
\hat{\Omega}=\left\{x \in \Omega: \hat{E} \neq \frac{1}{2}\left((I+D u)^{T}(I+D u)-I\right)\right\} .
$$

The argument is similar as that in lemma 2.4 (see also [8]).

The results in this section show that, by solving the finite problem of minimizing $I_{M}(\cdot)$ in $A_{h}$ with $M$ sufficiently large and $h$ sufficiently small, we can obtain good information on the solutions of the problem of minimizing $I(\cdot)$ in $A$. For example, let $u_{M, h} \in A_{h}$ be a minimizer of $I_{M}(\cdot)$ in $A_{h}$ with $M$ sufficiently large and $h$ sufficiently small, if $u_{M, h}$ oscilates violently somewhere in $\Omega$, then it suggests that microstructure probably exists as a solution, on the other hand, if $u_{M, h}$ does not oscilate anywhere in $\Omega$, then it should give a good approximation to a minimizer of $I(\cdot)$ in $A$. 


\section{References}

[1] J.M. Ball, Convexity conditions and existence theorems in nonlinear elasticity. Arch. Rat. Mech. Anal., 63(1977), 337-403.

[2] J.M. Ball, Constitutive inequalities and existence theorems in nonlinear elastostatics, in Nonlinear Analysis and Mechanics: Heriot-Watt Symposium Vol.1 (edited by R.J. Knops), Pitman, London, 1977.

[3] Z.-P. Li, The finite element method for boundary value problems of nonlinear three-dimensional elasticity. Northeastern Math. J., 2(1986), 449-467.

[4] Z.-P. Li, Existence theorem and finite element method for static problems of a class of nonlinear hyperelastic shells. Chin. Ann. of Math., 10B(1989), 169-189.

[5] P.V. Negròn Marrero, A numerical method for detecting singular minimizers of multidimensional problems in nonlinear elasticity. Numer. Math., 58(1990), 135-144.

[6] Z.-P. Li, Element removal method for singular minimizers in problems of hyperelasticity. to appear in Math. Models and Methods in Appl. Sci., 3(5)(1995).

[7] P.G. Ciarlet, Mathemetical Elasticity Vol.1: Three-dimensional Elasticity, North-Holland, Amsterdam, 1988.

[8] Z.-P. Li, Existence of minimizers and microstructures in nonlinear elasticity. to appear in Nonlinear Anal.: Theo., Meth. Appl..

[9] J.M. Ball and J.E. Marsden, Quasiconvexity at the boundary, positivity of the second variation, and elastic stability, Arch. Rat. Mech. Anal., 86(1984), 251-277.

[10] P.G. Ciarlet, The Finite Element Method for Elliptic Problems. North-Holland, Amsterdam, 1978.

[11] Z.-P. Li, A numerical method for computing singular minimizers. to appear in Numer. Math..

[12] J.M. Ball and G. Knowles, A numerical method for detecting singular minimizers. Numer. Math., 51(1987), 181-197. 
[13] Z.-P. Li, Element removal method for singular minimizers in variational problems involving Lavrentiev phenomenon. Proc. R. Soc. Lond. A, 439(1992), 131-137.

[14] A.D. Ioffe, On lower semicontinuity of integral functionals, I and II. SIAM J. Control and Optimization, 15(1977), 521-538, 991-1000.

[15] R.A. Adams, Sobolev Spaces, Academic Press, New York, 1975.

[16] Z.-P. Li, A theorem on lower semicontinuity of integral functionals. to appear in Proc. R. Soc. Edinburgh. Seri. A. 